\title{
Effectiveness Simulation of Internal Heat Exchanger (IHX) Combination Investigation on $\mathrm{CO}_{2}$ Refrigeration
}

\author{
I Dewa M C Santosa ${ }^{1}$, IGN Suta Waisnawa ${ }^{2}$ I Wayan Temaja \\ Mechanical Engineering Department \\ Politeknik Negeri Bali \\ Bali, Indonesia \\ 1idmcsantosa@pnb.ac.id, 2sutawaisnawa@pnb.ac.id
}

\begin{abstract}
As natural refrigerant, carbon dioxide has excellent thermo physics, safe, nontoxic and cheap. This reason causes the $\mathrm{CO}_{2}$ becomes the best refrigerant alternatives. However, $\mathrm{CO}_{2}$ refrigeration system still has relatively low performance when operate in hot environment temperature. This study aims is to investigate the performance of $\mathrm{CO}_{2}$ trans critical refrigeration system using Internal Heat Exchanger (IHX) in tropical outdoor temperature and will compare with standard $\mathrm{CO}_{2}$ refrigeration system which has been studied previously. The research will be carried out by theoretical study and numerical analysis of the refrigeration system using the EES (Engineering Equation Solver) program. Data input and simulation validation are obtained from experimental and secondary data. The result shows that the simulation has good agreement with the experimental results. Errors only as much as $1 \%$ up to $2 \%$ so it can be argued that the simulation valid for $\mathrm{CO}_{2}$ refrigeration system with IHX combination analysis especially when the system is operated in tropical climate i.e. Indonesia.
\end{abstract}

Keywords- $\mathrm{CO}_{2}$ refrigeration system, Performance (COP), tropical climate, Internal Heat Exchanger (IHX)

\section{INTRODUCTION}

The study of vapor compression systems of $\mathrm{CO}_{2}$ refrigeration systems were carried out by many researchers based on various climate conditions in the world. For tropical climates (hot climate) this system will work in supercritical conditions due to high ambient temperatures. Data from previous studies obtained switch points from sub critical to trans critical in the $\mathrm{CO}_{2}$ Refrigeration system with a booster hot gas bypass with an optimum gas cooler component, occurring at temperatures above $27^{\circ} \mathrm{C}$ [1] and of course this condition changes according to the performance of the gas cooler, where this point will be higher when the performance of the gas cooler become better. To get the optimum gas cooler performance, the correlation between ambient temperature and the gas cooler optimum pressure can produce the highest Coefficient of Performance.

It is found that COP varies with ambient temperature and work pressure, the higher the external environment temperature, the lower the COP, so it is very clear that application to high temperature environments requires design modifications to increase the COP of the system [2]. Tsamos et al. [3] examined several system configurations to be tested at ambient temperatures (warm climate). The results obtained show that a $\mathrm{CO}_{2}$ booster system with hot gas bypass can provide the best performance with $5.0 \%$ energy savings for warm climates and $3.65 \%$ for moderate climates, followed by an integrated cascade of all $\mathrm{CO}_{2}$ systems with gas bypass compressors, with $3,6 \%$ and $2.1 \%$ savings on the reference system for each warm and moderate climate. . In line with this research, so that for tropical regions needs to be studied in detail to get optimal performance. Optimum pressure characteristics in trans critical operating conditions, that in order to get the maximum COP at each increase in environmental conditions, the operation of the system at optimum pressure is also higher [4].

Studies on the application of internal heat exchanger methods have been carried out with various combinations and different environmental conditions. Sharma et al. [5] examined several configurations of the R404A multiplex direct expansion system with a $\mathrm{CO}_{2}$ system combining booster and cascade / secondary loop systems to be applied in several climate zones in the United States to get the maximum COP in each of these climate zones. It was found that various $\mathrm{CO}_{2}$ system design configurations for supermarket applications have performance optimizations in each of the different climate zones. This is similar to Pérez et al. [6] which results in that certain design configurations can only be efficient in conditions also, for example, for a $\mathrm{T}_{\text {out }}$ (temperature of gas cooler outlet from $31^{\circ} \mathrm{C}$ ) it is not recommended to use an internal heat exchanger (IHX) although from all design configurations it is found that adding an internal heat exchanger can change the COP. Sánchez et al. [7] conducted an experimental study on the implementation of the IHX system. It was found that the COP increase was around $13 \%$ in optimal conditions. Nakagawa et al. [8] developed a two-phase ejector with internal heat exchanger (IHX) and based on experimental results obtained significantly improved the performance coefficient (COP) of the ejector system. With the specific conditions that have been used, the ejector system with IHX can increase the COP system by $27 \%$ 
compared to the same conventional system. By increasing the degree of sub cooling in the $\mathrm{CO}_{2}$ refrigeration system also shows a significant effect on performance (COP). Where, review is limited to $\mathrm{CO}_{2}$ refrigeration cycles with accumulation of receivers for commercial purposes and does not consider air conditioning or MAC systems. Improved system performance generally found that with internal heat exchangers improvements up to $12 \%$, with economizers, with thermoelectric systems $25.6 \%$ and with sub-cooling methods $30.3 \%$ [9-11].

Thus the aim of this research is to provide a suitable simulation to analyze the characteristics of the Coefficient of Performance (COP) of the $\mathrm{CO}_{2}$ Refrigeration System with the Hot Gas Bypass Booster System which is applied to supermarkets with Indonesia's climate with the principles of Thermodynamic Law. Secondly, is to study the increase (COP) of $\mathrm{CO}_{2}$ refrigeration systems with the addition of internal heat exchanger (IHX) combinations. And as far as researchers know, none of the studies that have made various internal heat exchanger configurations for $\mathrm{COP}$ enhancements from $\mathrm{CO}_{2}$ booster bypass refrigeration systems to be applied in Indonesia.

\section{RESEARCH METHODOLOGY}

\section{A. Sistem Design}

This study was carried out with theoretical studies and numerical analysis of vapor compression refrigeration systems using the EES (Engineering Equation Solver) program [12]. Equations are developed from Thermodynamic Laws I and II supported by survey data and secondary data as input and for validation modeling. The system under study is the Trans critical $\mathrm{CO}_{2}$ Refrigeration System with Hot gas Bypass Booster for supermarket applications, as shown in Figure 1.

This system is a two stage system so that it uses a low pressure compressor (LP) and high pressure compressor (HP) and at low pressure works the Low Temperature (LT) system with a temperature of up to $-30{ }^{\circ} \mathrm{C}$ which is used for the freezer (Evaporator-LT) and also works Medium Temperature (MT) systems with temperatures ranging from $15^{\circ} \mathrm{C}$ until $-10^{\circ} \mathrm{C}$ used as a chiller / fridge in supermarket applications.

The IHX combination is placed in positions in accordance with Figure 1. Internal heat exchanger (IHX) is combined among three het exchanger (IHX-1, IHX-2, and IHX-3). The position of IHX-3 is a combination of refrigerant out the gas cooler / condenser with a refrigerator that flows into the HT compressor, this is intended to obtain or reduce the degree of superheated refrigerant that enters the HT compressor (or also called an intercooler). The superheated condition of the compressor can be stabilized which affects the thermal efficiency of the compressor which is getting higher. The IHX2 position is in a combination of superheated from compressor discharge LT with sub-cooled liquid refrigerant in the reservoir. IHX -3 is placed in a position before the HT compressor so it can be assured that the condition of the incoming refrigerant is superheated.

\section{B. Research Step}

Firstly, this study begins with a survey of $\mathrm{CO}_{2}$ refrigeration systems that have been developed in Indonesia to obtain data on system performance. The system has been surveyed is $\mathrm{CO}_{2}$ refrigeration systems developed in the Department of Refrigeration and Air Conditioning Engineering, State Polytechnic of Bandung. This survey data will also be used as a comparative study for various refrigeration systems developed in Indonesia. For data requirements - data input and system validation will be used data - data from experiments, surveys and secondary data. The other experimental data are obtained from the data of collaboration with Brunel University, data

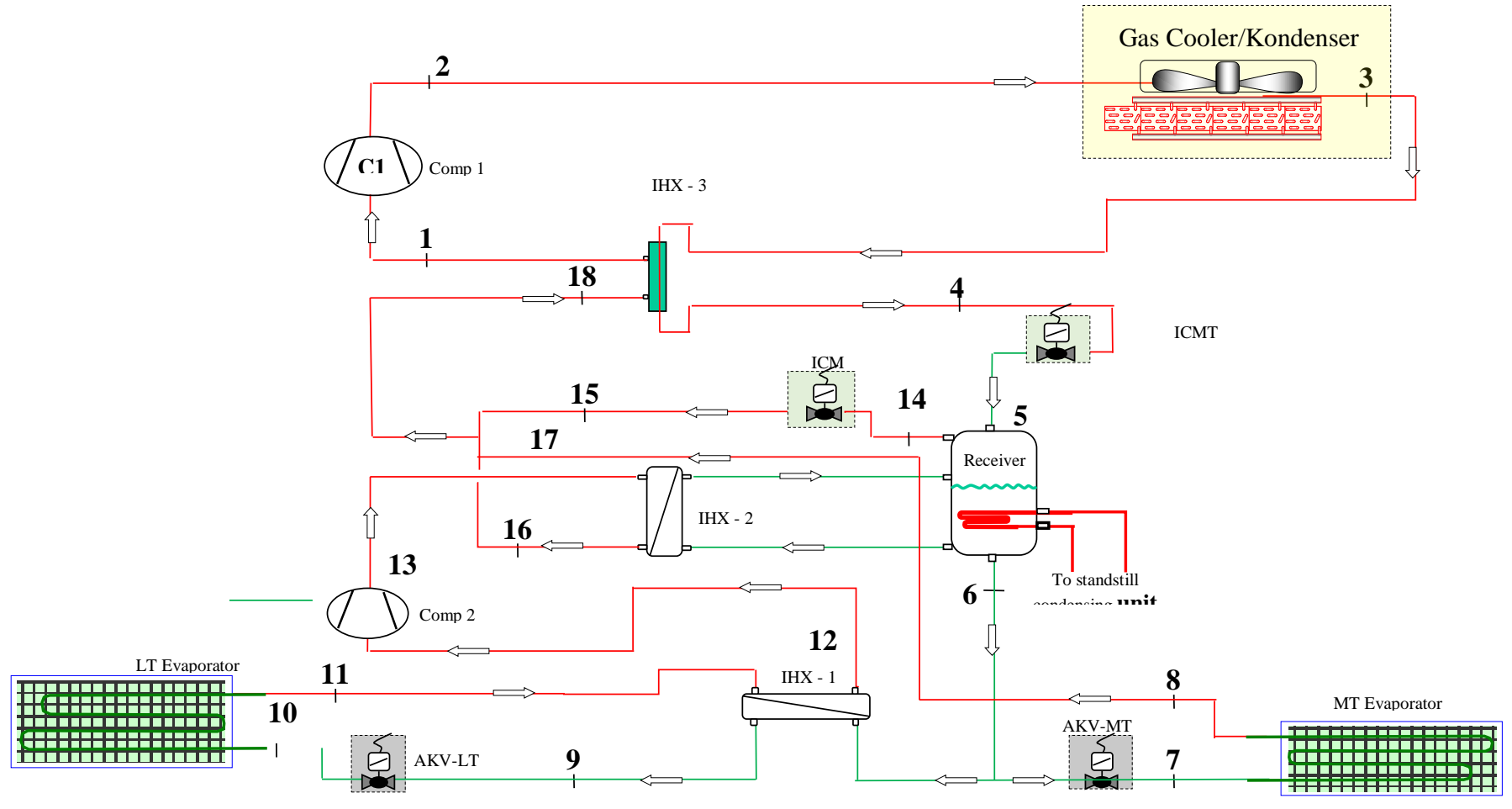

Fig. 1. The CO2 refrigeration system with IHX combination 
input survey data will be taken in the State Polytechnic of Bali, addressing daily and monthly temperature / humidity profiles as well as data on the cooling capacity requirements of several large supermarkets in the Bali Province Region. Secondary data is obtained from international journals that have been published.

Secondly, Simulation based Engineering Equation Solver (EES) program is built. The equation developed bas on thermodynamic concept and develop a P-h diagram based on the design as shown in Figure 1. Finally, the simulation is validated against experimental data. Simulation validation mainly depend on temperature condition in gas cooler area, because temperature in this area are vary with ambient temperature and high pressure as shown in Table1.

\section{RESULT AND DISCUSSIONS}

This preliminary result has been obtained from running programs built with EES and the program has obtained good results as evidenced by the validation of simulation results with experimental data for $\mathrm{CO}_{2}$ systems but only with medium temperature systems as shown in Figure 2.

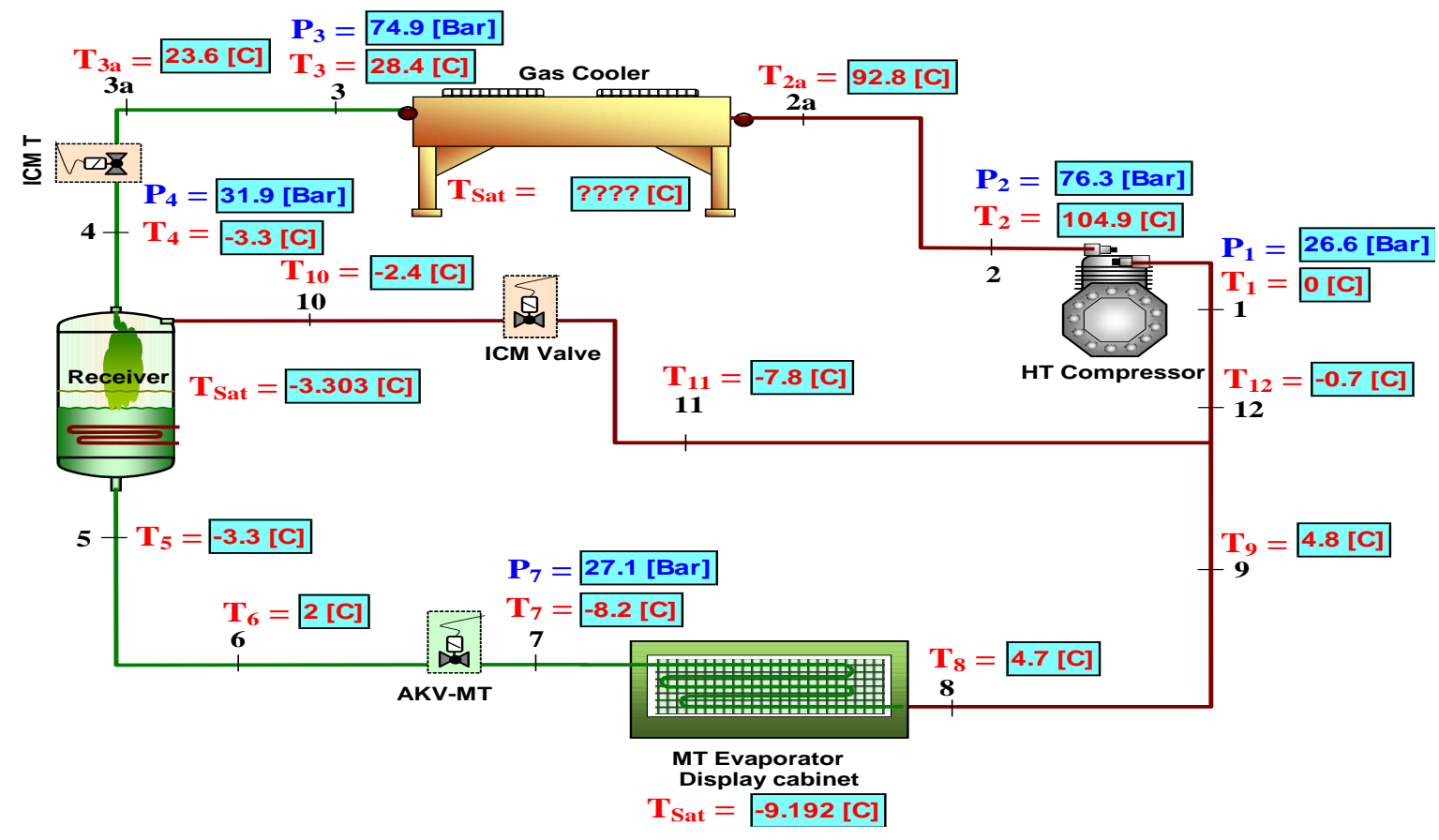

Fig.2. Data from experimetal test with specific condition

TABLE I. SIMULATION VALIDATION

\begin{tabular}{|c|c|c|c|c|c|c|c|c|c|}
\hline \multicolumn{4}{|c|}{ Data model (average) } & \multicolumn{4}{|c|}{ Data eksperiment (average) } & \multicolumn{2}{|c|}{ errors } \\
\hline P-high & Tamb & $\mathrm{T} 2$ & T3 & P-high & Tamb & $\mathrm{T} 2$ & T3 & $\mathrm{T} 2$ & T3 \\
\hline (Кра) & $(\operatorname{deg} C)$ & $(\operatorname{deg} C)$ & $(\operatorname{deg} C)$ & (Кра) & $(\operatorname{deg} C)$ & $(\operatorname{deg} C)$ & $(\operatorname{deg} C)$ & $(\%)$ & (\%) \\
\hline 7.528 & 25 & 103,2 & 28 & 7.528 & 25 & 104,9 & 28,45 & $2 \%$ & $2 \%$ \\
\hline 8.213 & 28 & 113,5 & 31 & 8.213 & 28 & 114,3 & 31,35 & $1 \%$ & $1 \%$ \\
\hline 8.342 & 29 & 115,7 & 32 & 8.342 & 29 & 115,2 & 31,77 & $0 \%$ & $-1 \%$ \\
\hline 8.526 & 30 & 118,5 & 33 & 8.526 & 30 & 116,9 & 33,24 & $-1 \%$ & $1 \%$ \\
\hline
\end{tabular}

An important system to be validated according to the experimental data obtained is $\mathrm{T} 2$ and $\mathrm{T} 3$, others can be controlled according to the best control conditions. Intermediate pressure, high pressure, medium temperature and low pressure can be adjusted according to the desired conditions.

Validation is carried out by analyzing in detail the results of the experiments that were collaborated with the Brunel University that had been done previously. The following is shown the data obtained from the results of the experiment to determine the degree of accuracy of the resulting simulation, an example of the experiment that works with Brunel University from the validation obtained that there was an error of $1 \%$ up to $2 \%$ and based on EES simulation (see Table 1), a valid simulation has been obtained. Because error from a large system with a high temperature (high difference). Thus this simulation is valid enough to analyze the $\mathrm{CO}_{2}$ system that will be carried out in previous research, by including variations in environmental temperature and high pressure variations, and will be compared with previous research in detail.

Compared to standard designs (without internal heat exchangers) that have been studied previously, the design consists of 14 state points $[2,13]$ with sub cooled degrees in the saturated position (without sub-cooled). Previous studies also show that sub cooling degrees are very significant to improve the performance of $\mathrm{CO}_{2}$ refrigeration systems up to $30 \%$ [911]. From the development of the $\mathrm{CO}_{2}$ system design with IHX combination, the state point was developed into 18 points, the results of EES development are shown in Figure 3. 
According to the preliminary data, the system simulation results obtained a P-h diagram where in general it is obtained an increase in the degree of sub-cooled and superheated degrees from the system so that the expected performance (COP) can be increased which will be explored in depth in the next research.

\section{CONCLUSION}

The simulation results that have been generated show a fairly good validation of $1 \%-2 \%$ of the results against the experiment results. The error rate shows high confident results because the system designed is a large system with very high temperature differences at each point. It can be argued that this simulation is very good for analyzing $\mathrm{CO}_{2}$ Refrigeration systems with IHX combinations as well as with other development methods. So that the simulation can replace the experimental test which is expensive and requires extra time, so that it can be further analyzed the optimum conditions to be operated in tropical regions such as Indonesia. The effect of IHX is generally seen on the increase in sub cooling or superheated, thus the internal heat exchanger and its combination will be obtained to have a positive effect on the overall system performance on systems that are operated in hot (tropical) environments.

\section{ACKNOWLEDGMENT}

This study was fully supported by the DRPM RISTEK DIKTI, Ministry of Research, Technology and Higher Education -Indonesian Government, Funding No: SP Dipa042.06.1.401516/2018 for the in cash contribution. The authors wish to acknowledge the contributions of Mechanical Engineering Department -Bali State Polytechnic for the in-kind contributions and CSEF Brunel University for share $\mathrm{CO}_{2}$ refrigeration system data. Also Center for Research and Community Service (P3M) for all administrative support.

\section{REFERENCES}

[1] I.D.M.C. Santosa, B.L. Gowreesunker, S.A. Tassou, and K.M. Tsamos, "Investigations into air and refrigerant side heat transfer coefficients of finned-tube $\mathrm{CO}_{2}$ gas coolers". Int. J. Heat. \& Mass Transfer, vol. 107, pp. 168-180, 2017.

[2] I.D.M.C. Santosa, Sudirman, and I.N.G.S. Waisnawa, "Performance Analysis of Transcritical $\mathrm{CO}_{2}$ Refrigeration System for Supermarket Application", Int. J. of Geomate, Vol.15, Issue 50, pp. 70 - 75. 2018

[3] K.M.Tsamosa, Y.T. Ge. I.D.M.C. Santosa, S.A. Tassoua, G. Bianchia, and Z. Mylona, "Energy analysis of alternative $\mathrm{CO}_{2}$ refrigeration system configurations for retail food applications in moderate and warm climates", Energy Conversion and Management, vol. 150, pp. 822-829, 2017.

[4] Y.T. Ge and S.A. Tassou, "Thermodynamic analysis of transcritical $\mathrm{CO}_{2}$ booster refrigeration systems in supermarket. Energy Convers. and Management, vol. 52, pp. 1868-1875, 2011

[5] V.D. Sharma, B. Fricke, P. Bansal, Comparative analysis of various $\mathrm{CO}_{2}$ configurations in supermarket refrigeration systems, Intl. J. Refrigeration, vol. 46, pp. 86-99, 2014.

[6] G.V. Pérez, F.J.M Belman., E.J. Navarro, and M.C. Rubio. "Comparative study of transcritical vapor compression configurations using $\mathrm{CO}_{2}$ as refrigeration mode base on simulation". App. Therm. Eng., vol. 51, pp. 1038-1046. 2013

[7] D. Sánchez, J. Patiño, R. Llopis, R. Cabello, E. Torrella, and F.V. Fuentes, "New positions for an internal heat exchanger in a $\mathrm{CO}_{2}$ supercritical refrigeration plant", Experimental analysis and energetic evaluation, Appl. Therm. Eng., vol 63, pp. 129-139, 2014

[8] M. Nakagawa, A.R. Marasigan, T. Matsukawa, "Experimental analysis on the effect of internal heat exchanger in transcritical $\mathrm{CO}_{2}$ refrigeration cycle with two-phase ejector", Intl. J. of Refrigeration, vol.34, pp. 15771586. 2011

[9] Rodrigo Llopis, Laura Nebot-Andrés, Daniel Sánchez, Jesús CatalánGil, and R. Cabello, "Sub cooling methods for $\mathrm{CO}_{2}$ refrigeration cycles". A Review. Intl. J. of Refrigeration 41,xxx-xxx. 2018

[10] Y.B. Tao, Y.L. He, and W.Q. Tao, "Exergetic analysis of transcritical $\mathrm{CO}_{2}$ residential air-conditioning system based on experimental data", Appl. Energy, vol. 87, pp. 3065-3072. 2010

[11] E. Torrella, D. Sa'nchez, R. Llopis, and R. Cabello, "Energetic evaluation of an internal heat exchanger in a $\mathrm{CO}_{2}$ trans critical refrigeration plant using experimental data", Intl. J. of Refrigeration vol. 34, pp.40-49. 2011.

[12] F-Chart Software. EES (engineering equation solver); 2017.<http://www.fchart.com>.

[13] I.D.M.C. Santosa, Sudirman, I.G.N.S. Waisnawa, P.W. Sunu, and I.W. Temaja, "Simulation of transcritical $\mathrm{CO}_{2}$ refrigeration system with booster hot gas bypass in tropical climate". J. of Phys.: Conf. Series. Vol. 953, pp. 012044. 2018 